Letter

\section{A Case of Scleromyxedema Development Following Intravesical Bacillus Calmette-Guérin Administration}

\section{To the Editor:}

We read with great interest the case report written by Boleto et $\mathrm{al}^{1}$ entitled, "Rapidly Progressive Systemic Sclerosis-associated Interstitial Lung Disease After Intravesical Bacillus Calmette-Guérin Therapy for Early-stage Bladder Cancer". In this article, the authors speculated on a possible association between intravesical bacillus Calmette-Guérin (BCG) administration and progression in systemic sclerosis (SSc)-associated interstitial lung disease. Our aim is to present a case that will support the authors' theory indirectly, as well as to briefly discuss the possible pathogenesis in the context of intravesical BCG-mediated SSc, thereby increasing the necessary awareness of this issue among physicians.

A 48-year-old male with a previous history of hypertension and bladder cancer was admitted due to the development of progressive widespread skin tightness and decreased movement in all extremities. The patient's medical history revealed that 6 weekly intravesical BCG instillations following transurethral resection (TUR) was commenced in 2017. After 3 years in remission, a second TUR was performed, together with a 6-week BCG treatment due to tumor recurrence. Two weeks after the last BCG administration, rapid stiffness and inability to move developed first in the left forearm and subsequently in the other extremities. The patient was referred to our department for further evaluation and treatment. Informed consent was obtained from the patient. Ethical approval for this case was waived because no ethical approval for the publication of case reports is demanded by local ethics committee of Eskişehir Osmangazi University.

Physical examination showed an immobile, middle-aged male with increased skin stiffness (modified Rodnan skin score [mRss] 34/51), decreased range of motion, and pain in all extremities. The metacarpophalangeal and proximal interphalangeal joints of the left hand were painful and swollen. Cardiac and lung examinations were noncontributory. Laboratory investigations showed normal levels of erythrocyte sedimentation rate $(25 \mathrm{~mm} / \mathrm{h})$, C-reactive protein $(4.5 \mathrm{mg} / \mathrm{dL}$; normal range $0-5$ ), and creatine kinase (77 U/L; normal range 46-195), but partially elevated levels of aspartate aminotransferase ( $58 \mathrm{U} / \mathrm{L}$; normal range $0-37$ ), alanine aminotransferase ( $56 \mathrm{U} / \mathrm{L}$; normal range 0-37), and lactate dehydrogenase ( $493 \mathrm{U} / \mathrm{L}$; normal range 135-225). Serum thyroid-stimulating hormone level was normal and serum antinuclear antibody was negative. Skin biopsy evaluation demonstrated fibrosis in the dermis and subcutaneous fat tissue, and separation of increased collagen fibers together with dense mucin formation, suggesting scleromyxedema. Magnetic resonance imaging of lower extremities was remarkable for increased heterogeneous signals within all muscle compartments and liquid deposition to the dermis and subcutaneous fat tissue. According to clinical and histopathologic findings, diagnosis of BCG-related scleromyxedema was considered. No evidence of any internal organ involvement was detected based on extensive evaluation. Serum protein electrophoresis was normal, and malignancy examination was nonrevealing. The patient was initially placed on combination of steroids $(1 \mathrm{mg} / \mathrm{kg} / \mathrm{d}$ methylprednisolone) and methotrexate (MTX; $15 \mathrm{mg}$ subcutaneous per week) in conjunction with physical rehabilitation during this hospital stay. At the first month follow-up, due to inadequate improvement regarding skin findings and mobility of extremities as well as development of MTX-related moderate elevation in liver function tests, intravenous Ig (IVIG) therapy $(0.5 \mathrm{~g} / \mathrm{kg} / \mathrm{d})$ was instituted, with referral to rehabilitation thereafter. At admission for the second dose of IVIG, physical examination revealed a mobile patient with increased range of motion in all extremities and significant improvement in skin induration $(\mathrm{mRSS}=14 / 51)$.

Boleto et $\mathrm{al}^{1}$ reported a case with previous diagnosis of SSc, in which skin features did not progress with the initiation of BCG. One localized form of fibrosis leading to bladder contracture after BCG therapy has been previously reported in the literature. ${ }^{2}$ To our knowledge, our case is the first to establish the development of scleromyxedema following intravesical BCG. At admission, we initially considered the possibilities of scleroderma-like disorders secondary to malignancy and/or, less commonly, BCG use. However, no evidence of malignancy despite extensive investigations shows BCG as the inciting factor in this case.

Scleromyxedema is a type of scleroderma-like disorders primarily characterized by diffuse mucin and collagen depositions, and proliferation of fibroblasts. ${ }^{3}$ Although the pathogenesis has not been elucidated so far, some cytokines such as tumor necrosis factor and interleukin-1 have been proposed to play a role in the upregulation of hyaluronic acid synthesis by activating human fibroblasts. ${ }^{4}$ On the other hand, as it is generally associated with underlying monoclonal gammopathy, some experts have also put forward the debatable role of paraproteins.

BCG is an established effective immunotherapy, complementary to TUR, in superficial bladder cancer with uncertain mechanisms. Despite its good tolerability, local (BCGitis) and/or systemic types of complications (BCGosis) such as disseminated infection, reactive arthritis, remitting seronegative symmetrical synovitis with pitting edema, mycotic aneurysms, and hepatitis may rarely emerge following BCG exposure. ${ }^{5,6,7}$ Interestingly, previous studies have revealed that the innate immune cells have their own memory capacities that produce an enhanced response when subjected to secondary stimulation. This is called trained immunity. ${ }^{8}$ It has been reported that there is a dual interaction of trained macrophages and fibroblasts. As a result of trained immunity, BCG-trained macrophages may lead to skin inflammation and fibrosis. 'The development of skin involve- 
ment in our case can be explained by these pathogenic mechanisms. We believe that our case will increase awareness of the immunological-driven side effects of BCG therapy, especially from a rheumatological perspective. Studies on this subject may lead to results that will shed light on the pathogenesis of SSc or scleroderma-like disorders.

\section{Reşit Yıldırım ${ }^{1}$, MD}

Döndü Üsküdar Cansu' (iD, MD, Professor

Cengiz Korkmaz ${ }^{1}$ ( $)$, MD, Professor

${ }^{1}$ Division of Rheumatology, Department of Internal Medicine, Eskişehir Osmangazi University, Eskişehir, Turkey.

The authors declare no conflicts of interest relevant to this article.

Address correspondence to Dr. R. Yildırım,

Department of Rheumatology, School of Medicine,

Eskişehir Osmangazi University Eskişehir, 26480, Turkey.

Email: celeng18@gmail.com.

\section{REFERENCES}

1. Boleto G, Avouac J, Allanore Y. Rapidly progressive systemic sclerosis-associated interstitial lung disease after intravesical bacillus Calmette-Guérin therapy for early-stage bladder cancer. J Rheumatol 2021;48:1348-1349.

2. Garcia C, Jina H, Bergersen P, Chalasani V. Bladder contracture - a rare and serious side effect of intravesical bacillus Calmette-Guérin therapy. Urol Case Rep 2015;4:22-4.
3. Ferreli C, Gasparini G, Parodi A, Cozzani E, Rongioletti F, Atzori L. Cutaneous manifestations of scleroderma and scleroderma-like disorders: a comprehensive review. Clin Rev Allergy Immunol 2017;53:306-36.

4. Campo GM, Avenoso A, Campo S, Angela D, Ferlazzo AM, Calatroni A. TNF-alpha, IFN-gamma, and IL-1beta modulate hyaluronan synthase expression in human skin fibroblasts: synergistic effect by concomital treatment with $\mathrm{FeSO} 4$ plus ascorbate. Mol Cell Biochem 2006;292:169-78.

5. Pérez-Jacoiste Asín MA, Fernández-Ruiz M, López-Medrano F, et al. Bacillus Calmette-Guérin (BCG) infection following intravesical BCG administration as adjunctive therapy for bladder cancer: incidence, risk factors, and outcome in a single-institution series and review of the literature. Medicine 2014;93:236-54.

6. Macleod LC, Ngo TC, Gonzalgo ML. Complications of intravesical bacillus Calmette-Guérin. Can Urol Assoc J 2014;8:E540-4.

7. Liu Y, Lu J, Huang Y, Ma L. Clinical spectrum of complications induced by intravesical immunotherapy of bacillus Calmette-Guérin for bladder cancer. J Oncol 2019:6230409.

8. Arts RJW, Moorlag SJCFM, Novakovic B, et al. BCG vaccination protects against experimental viral infection in humans through the induction of cytokines associated with trained immunity. Cell Host Microbe 2018;23:89-100.

9. Jeljeli M, Riccio LGC, Doridot L, et al. Trained immunity modulates inflammation-induced fibrosis. Nat Commun 2019;10:5670. 